\title{
Changes in Immunohistochemical Levels and Subcellular Localization After Therapy and Correlation and Colocalization With CD68 Suggest a Pathogenetic Role of Hsp60 in Ulcerative Colitis
}

\author{
Giovanni Tomasello, MD, PhD,* Vito Rodolico, MD, $\dagger$ Monica Zerilli, PhD, $\dagger$ Anna Martorana, MD, $\dagger$ \\ Fabio Bucchieri, MD, $\$$ Alessandro Pitruzzella, MSc, $\$$ \& Antonella Marino Gammazza, PhD, $\$$ \& \\ Sabrina David, MSc, $\$$ Francesca Rappa, MD, $\$$ Siovanni Zummo, MD, $\$$ Provvidenza Damiani, MD,\| \\ Salvatore Accomando, MD,\$ף Manfredi Rizzo, MD,\# Everly Conway de Macario, PhD, ** \\ Alberto J.L. Macario, MD, $\S^{* *}$ and Francesco Cappello, MD $\sharp$
}

\begin{abstract}
In an earlier work, the role of heat shock protein (Hsp60) in the pathogenesis of ulcerative colitis (UC) was suggested by its significant increase in the pathological mucosa parallel with an increase in inflammatory cells. More data in this direction are reported in this work. We analyzed by immunohistochemistry biopsies of colon tissue from 2 groups of patients with UC and treated with either 5-aminosalicylic acid (5-ASA) alone or in combination with a probiotic. We looked for inflammatory markers and Hsp60. Both the treatments were effective in reducing symptoms but the group treated with both 5-ASA and probiotics showed better clinical results. Amelioration of symptoms was associated with reduction of both inflammation and Hsp60, a reduction that was most marked in the group treated with 5-ASA and probiotics. The levels of Hsp60 positively correlated with those of CD68-positive cells, and double immunofluorescence showed a high index of colocalization of the chaperonin and CD68 in lamina propria. Immunoelectron microscopy showed that
\end{abstract}

Received for publication October 12, 2010; accepted January 20, 2011. From the *Dipartimento di Chirurgia Generale, d'Urgenza e Trapianti d'Organo; †Dipartimento di Patologia Umana; $\$$ Dipartimento di Biomedicina Sperimentale e Neuroscienze Cliniche; $\|$ Dipartimento di Medicina Interna, Malattie Cardiovascolari e Nefrourologiche; -Dipartimento Materno-Infantile; \#Dipartimento di Medicina Interna e delle Malattie Emergenti, Università degli Studi di Palermo; §Istituto Euro-Mediterraneo di Scienza e Tecnologia (IEMEST), Palermo, Italy; and **Department of Microbiology and Immunology, School of Medicine, and IMET, University of Maryland, Baltimore, MD.

Supported by funds from MIUR ex-60\% (V.R., G.Z., F.B., and F.C.) and from Istituto Euro-Mediterraneo di Scienza e Tecnologia (A.J.L.M., and F.C.). The funders had no role in study design, data collection and analysis, decision to publish, or preparation of the manuscript.

Giovanni Tomasello and Vito Rodolico have contributed equally to the present work.

The authors have declared that no competing interests exist.

Reprints: Francesco Cappello, MD, Dipartimento di Biomedicina Sperimentale e Neuroscienze Cliniche, Sezione di Anatomia Umana, via del Vespro 129, 90127, Palermo, Italy (e-mail: francapp@, hotmail.com).

Copyright (C) 2011 by Lippincott Williams \& Wilkins
Hsp60 - classically a mitochondrial protein - was abundantly also present in cytosol in biopsies taken at the time of diagnosis, but not after the treatment. Our data suggest that Hsp60 is an active player in pathogenesis of UC and it can be hypothesized that the chaperonin is responsible, at least in part, for initiation and maintenance of disease.

Key Words: Hsp60, chaperonin, ulcerative colitis, macrophages, CD68, inflammation, innate immunity

(Appl Immunohistochem Mol Morphol 2011;00:000-000)

$\mathrm{H}$ eat shock proteins (Hsps) are a large family of molecules highly conserved during evolution and, thus, are present in all living species, from archaea and bacteria to humans. ${ }^{1}$ This is probably owing to the fact that stress is an ancestral phenomenon and primordial cells had the need to develop antistress proteins like Hsps. Many Hsps are also chaperones and they are involved in folding, refolding, translocation, and degradation of intracellular proteins, both under normal and stress conditions. ${ }^{2}$ Despite the fact that not all Hsps are chaperones and, conversely, not all chaperones are Hsps, the terms Hsp and chaperone are used interchangeably in the literature, and we will do so here.

The importance of $\mathrm{Hsps} /$ chaperones in protein homeostasis and other cellular processes such as innate immunity is well documented and, therefore, their quantitative or qualitative alterations owing to, for instance, mutation, aberrant posttranslational modification, or gene dysregulation, can cause a variety of diseases now known as chaperonopathies. ${ }^{3}$ Further proof of the importance of Hsps/chaperones is that they can be considered therapeutic agents in replacement therapy (chaperonotherapy) or as targets for inhibition if they play a pathogenetic role. ${ }^{4}$ Probable examples of the latter case, that is, chaperones as agents of disease rather than protection against it, are inflammatory bowel diseases (IBDs). For example, we found that Hsp60 and Hsp10 
are increased in the affected mucosa in Crohn disease and ulcerative colitis (UC). ${ }^{5}$

Hsp60 is among the most conserved Hsps/chaperones. ${ }^{6}$ It has been classically described as a mitochondrial protein, ${ }^{7}$ although many studies have showed that it also resides outside mitochondria, for example in the cytosol, vesicles, and cell membrane. ${ }^{8,9}$ The presence and integrity of Hsp60 in mitochondria are crucial for cell survival, ${ }^{10-12}$ and at least 2 serious human genetic chaperonopathies occur because of the mutation of the $h s p 60$ gene. ${ }^{13,14} \mathrm{In}$ addition, increased levels of Hsp60 are a hallmark of a number of neoplasms ${ }^{15}$ and autoimmune disorders. ${ }^{16,17}$ In some of these pathologies, not only Hsp60 overexpression has been documented, but also an unusual subcellular localization has been described ${ }^{18-21}$ and, consequently, a pathogenetic role for the chaperonin has been proposed.

We have previously found increased levels of Hsp60 in a number of human neoplasms, including large bowel carcinomas, and these levels were correlated with the stage and grade of the disease. ${ }^{22,23}$ As mentioned above, more recently we studied a series of patients with IBD - a condition considered high-risk for cancer development - and we found increased levels of Hsp60 in both Crohn disease and UC, postulating that this protein could be implicated in their pathogenesis by triggering and/or maintaining inflammation. ${ }^{5}$ We found that the increased levels of the chaperonins were associated with an increase in inflammatory cells in both pathologies by comparison with normal mucosa. We also found that Hsp60 and Hsp10 occurred in the cytoplasm of mucosal epithelial cells in the 2 diseases but not in nonaffected tissue from controls. These findings, together with data from many laboratories demonstrating a role of chaperones in the etiology and pathogenesis of various diseases, as briefly mentioned in the initial paragraphs of this section, indicated that Hsp60 and Hsp10 deserve careful scrutiny as possible IBD causative agents. With this in mind, we proceeded to explore more in depth the possible participation of chaperonins in the initiation, progression, and relapse of IBD. For this purpose, we focused on 1 disease, UC, and on 1 chaperonin, Hsp60. It was important to confirm the increase in Hsp60 in diseased mucosa of UC and to determine whether the chaperonin would associate with cells that initiate the innate immune response, chiefly inflammatory cells, or with cells involved in the adaptive immune response, chiefly antibody production. Furthermore, it was important to establish if treatments that bring about clinical remission of UC also have an influence on Hsp60 and its associated immune response cells, innate or adaptive. A parallelism between clinical remission with decrease in the chaperonin levels and inflammation in the affected tissue would strongly support the notion that Hsp60 can contribute to disease and, also, that Hsp60 has to be seriously considered as a pathogenetic molecule in UC under certain circumstances, for example when it gains the extracellular space and interacts with inflammatory cells.

We report here the results of this detailed analysis of Hsp60 and inflammatory cells in the colon mucosa of patients with UC.

\section{MATERIALS AND METHODS}

\section{Patients}

We studied 40 patients with mildly to moderately active UC. Disease activity was assessed using the Mayo Score system from 0 to $12 .{ }^{24}$ Mildly to moderately disease activity was defined by a score of 4 to 10 . In these patients, we performed a randomized clinical study to compare the efficacy of the treatment for 6 months with 1.2 or $2.4 \mathrm{~g} / \mathrm{d}$ (depending on the disease severity) of mesalazine or 5aminosalicylic acid (5-ASA, Asacol, Giuliani, Italy) to that of the treatment with both 5-ASA and 1 capsule/day of probiotics (Acronelle, Bromatec, Italy).

All patients underwent flexible colonoscopy at the time of diagnosis and again at the 6-month follow-up visit (end point). During both the occasions, biopsies were taken, formalin-fixed, and sent to the pathologist for routine diagnostic examination. A small sample from each specimen was fixed in Karnofsky solution for immunoelectron microscopy (see below). Informed consent was obtained from all patients at the time of biopsy and the study was approved by the Ethics Committee of the University Hospital of Palermo, Italy.

\section{Tissue Preparations}

Specimens for immunohistochemistry and double immunofluorescence analyses (see below) were paraffinembedded and 4 to $5 \mu \mathrm{m}$ sections were obtained from all cases. We also studied 20 specimens of formalin-fixed paraffin-embedded normal colon mucosa from archival tissue, as controls.

Samples for immunoelectron microscopy were embedded in London Resin-white (Electron Microscopy Sciences, Hatfield, PA) following the manufacturer's protocols, ultracut with an ultramicrotome (Reichert Ultracut E), and mounted on 150-mesh uncoated gold grids for immunogold labeling (see below).

\section{Immunohistochemistry}

Immunostaining was done on 4 to $5 \mu \mathrm{m}$ tissue sections with a battery of antibodies, which are listed in Table 1, using an avidin-biotin complex kit (LSAB2, DAKO, Carpinteria, CA). Appropriate positive controls, as well as nonimmune serum for negative controls, were run concurrently. 3-3'-diaminobenzidine (DAB chromogen solution, DAKO) was used as developer chromogen. Nuclear counterstaining was done using hematoxylin (DAKO).

Three independent observers (F.C., M.Z., and A.M.) examined the specimens in a blind (code marked) approach and performed a quantitative analysis to determine: (a) the percentage of cells positive for the leukocyte markers CD3, CD4, CD8, CD20, and CD68 in colon lamina propria, and (b) the percentage of cells positive for Hsp60 both in epithelium and lamina propria of colon mucosa. All the observations were made at a magnification of $400 \times$ and the means of triplicate counts were used for statistical analyses. 


\begin{tabular}{|c|c|c|c|c|}
\hline $\begin{array}{l}\text { Antibody } \\
\text { Specific } \\
\text { for: }\end{array}$ & Supplier & $\begin{array}{l}\text { Catalog } \\
\text { Number }\end{array}$ & Source & Dilution \\
\hline Hsp60 & SIGMA & H4149 & Mouse & $1: 300^{*}$ \\
\hline Hsp60 & SIGMA & H4149 & Mouse & $1: 30 \dagger$ \\
\hline Hsp60 & StressGen & SPA-805 & Rabbit & $1: 100 \ddagger$ \\
\hline CD3 & Novocastra & $\begin{array}{l}\text { NCL-L- } \\
\text { CD3-PS1 }\end{array}$ & Mouse & $1: 100$ \\
\hline CD4 & Novocastra & $\begin{array}{l}\text { NCL-L- } \\
\text { CD4-1F6 }\end{array}$ & Mouse & $1: 100$ \\
\hline CD8 & Novocastra & $\begin{array}{l}\text { NCL-L- } \\
\text { CD8-295 }\end{array}$ & Mouse & $1: 100$ \\
\hline CD20 & Novocastra & $\begin{array}{l}\text { NCL-L- } \\
\text { CD20-L26 }\end{array}$ & Mouse & $1: 100$ \\
\hline CD68 & Novocastra & $\begin{array}{c}\text { NCL-CD68- } \\
\text { KP1 }\end{array}$ & Mouse & $1: 100$ \\
\hline
\end{tabular}

*For immunohistochemistry.

$\dagger$ For immunoelectron microscopy.

†For double immunofluorescence microscopy.

\section{Double Immunofluorescence}

Tissue sections were dewaxed in xylene, rehydrated in ethanol, washed in phosphate buffer solution (PBS), incubated with unmasking solution (tri-sodium citrate $10 \mathrm{mM}, 0.05 \%$ tween 20 ) for $10 \mathrm{~min}$ at $58^{\circ} \mathrm{C}$, and treated with blocking solution ( $3 \%$ bovine serum albumin in PBS) for 30 minutes at $24^{\circ} \mathrm{C}$. Then, the sections were incubated with the first primary antibody (mouse antiCD68; see Table 1 for details) overnight at $4^{\circ} \mathrm{C}$. The following day, some sections were incubated with the second primary antibody (rabbit anti-Hsp60; see Table 1 for details) overnight at $4^{\circ} \mathrm{C}$. After the incubations with the antibodies, the sections were washed 2 times in PBS, and were incubated with fluorescent secondary antibodies: mouse IgG antibody conjugated with fluorescein isothiocyanate (Sigma-Aldrich, Inc, Milan, Italy) and/or rabbit IgG antibody conjugated with Texas Red (Gene Tex Inc, Irvine, CA) for 1 hour at $24^{\circ} \mathrm{C}$. The nuclei were counterstained with Hoechst (Sigma-Aldrich, Inc, Milan, Italy) for 15 minutes at $24^{\circ} \mathrm{C}$. Finally, the sections were covered with a drop of PBS, the slides were mounted with cover slips, and readings and imaging were immediately performed with a Leica DM5000 upright fluorescence microscope (Leica Microsystems, Heidelberg, Germany). Two independent observers (F.C., and A.M.G.) examined the specimens in a blind (code marked) approach and performed a quantitative analysis to evaluate the number of cells that showed colocalization of CD68 and Hsp60 in lamina propria. All the observations were made at a magnification of $400 \times$ and the means of the duplicate counts were used for statistical analyses.

\section{Statistical Analysis}

Data obtained from immunohistochemistry and immunofluorescence evaluations were plotted using Microsoft Excel software (Microsoft Italia, Milan, Italy). Statistical analyses were carried out using the GraphPad Prism 4.0 package (GraphPad Inc, San Diego, CA) and

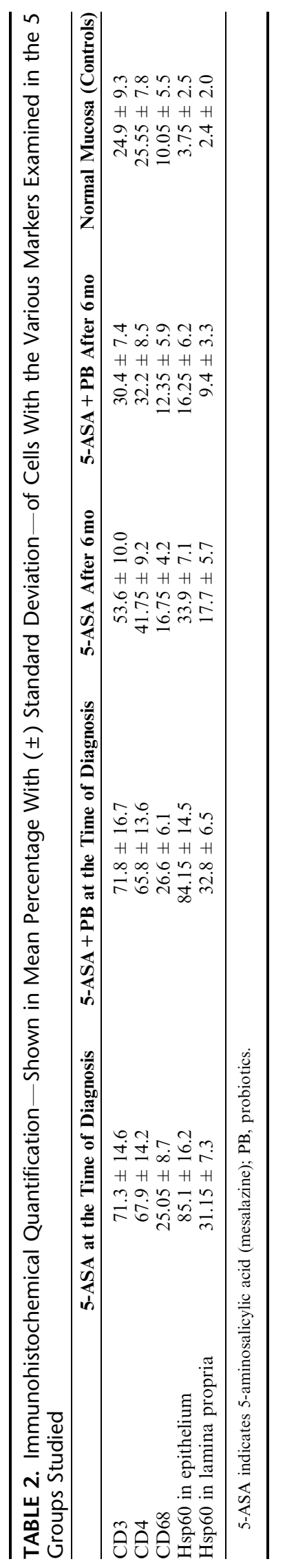

www.appliedimmunohist.com | 3 


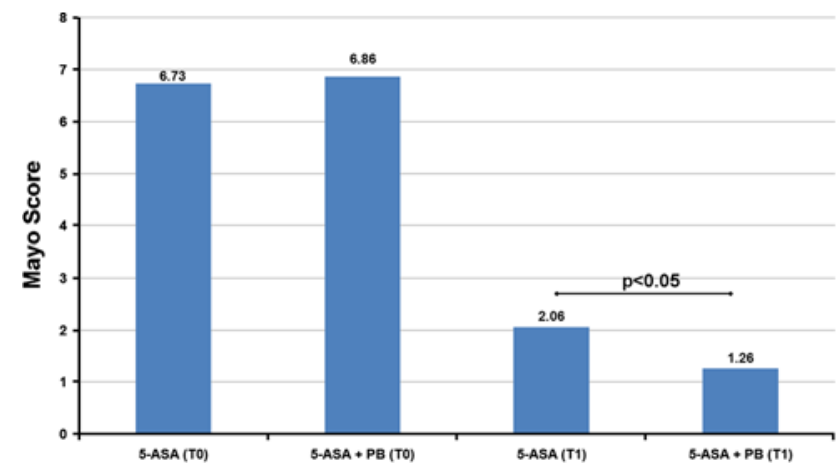

FIGURE 1. Clinical results. The bars show the Mayo Score of the 2 groups of patients - treated with mesalazine only (5-ASA) or with both 5-ASA and probiotics (PB) - at the time of diagnosis (TO) and at the 6-month end point (T1). Both treatments gave a significant reduction of the score (see text). Noteworthy is that the score after 5-ASA+PB treatment was significantly lower $(P<0.05)$ than after treatment with 5-ASA alone indicating that the treatment with the drug-probiotics combination was more efficacious than the drug alone. $\frac{\text { full color }}{0 \ln i n \mathrm{e}}$

nonparametric tests to compare groups that did not fit to the normal distribution. To evaluate the significance of differences between groups, the Wilcoxon test was used. Correlation analysis between $\mathrm{Hsp} 60$ and inflammatory markers was performed using the Pearson test, which gives a correlation coefficient (Pearson " $r$ ") and a " $p$ " value that measures the significance of the analyzed correlation. For correlation graphs, linear regression was also calculated to reflect the type of correlation between the chosen variables. Data were considered significant at an $\alpha$ level of $5 \%$. To have an indication on the variability of immunoreactivity data obtained by the different observers, the coefficient of variation was calculated using both the interobserver and intraobserver $\kappa$ statistics.

\section{Immunoelectron Microscopy}

London Resin-white resin embedding combined with postembedding immunogold labeling was used to localize Hsp60 within cells of colon mucosa by transmission electron microscopy. Briefly, a small fragment of colon tissue from patients who underwent flexible colonoscopy was excised from biopsy, cut into smaller pieces, postfixed with $0.5 \%$ glutaraldehyde $/ 2 \%$ paraformaldeyde (Karnofsky fixative) in sucrose-cacodylate buffer, and ultrathin sections were prepared as per standard procedures. Then, the ultrathin sections were treated with primary anti-Hsp60 antibody (see Table 1 for details) and secondary 10-nm gold-conjugated Aurion anti-mouse antibody (Electron Microscopy Sciences, Hatfield, PA) following the procedure described by others. ${ }^{8}$ Finally, the mesh were observed in a JEOL 1220 transmission electron microscope.

\section{RESULTS AND DISCUSSION}

\section{Clinical Results}

Patients with mild-to-moderate UC were randomized into 2 groups: one having patients treated only with 5-ASA and the other having patients treated with both 5ASA and probiotics (5-ASA $+\mathrm{PB})$. Both the treatments lasted for 6 months. Clinical results are summarized in Figure 1. At the time of diagnosis, both groups of patients had a Mayo Score between 4 and 10 (mean of 5-ASA patients: 6.73; mean of 5-ASA + PB patients: 6.86). After the treatment, both the groups showed a significant reduction $(P<0.001)$ in their scores (between 0 and 3; mean of 5-ASA patients: 2.06; mean of 5-ASA + PB patients: 1.26). In addition, the difference between the scores after the 2 treatments (2.06 vs. 1.26) was also significant $(P<0.05)$. Therefore, the combination 5-ASA + PB was more effective for score reduction than 5-ASA alone. These results are in agreement with other studies which showed that different combinations of probiotics are quite effective in ameliorating the symptoms of patients with UC. ${ }^{25-27}$

\section{Immunohistochemistry of Inflammatory Markers}

To assess the inflammatory cell component in all examined specimens, we used a panel of antibodies that
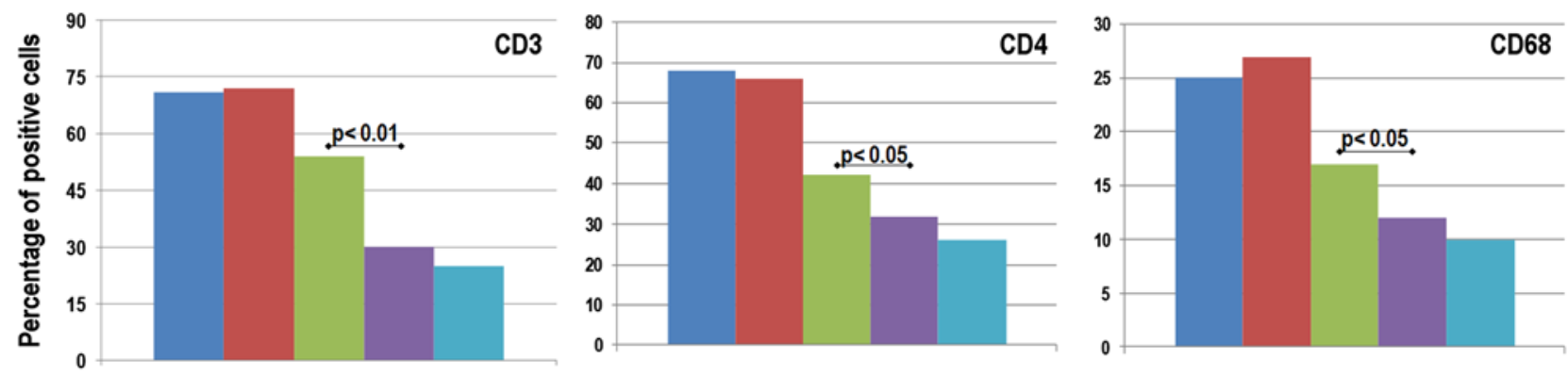

FIGURE 2. Immunohistochemical results for inflammatory markers. The bars show the levels of CD3, CD4, and CD68 in biopsies from patients before and after treatment with either 5-ASA alone or with the combination 5-ASA+PB. Both treatments produced a significant reduction $(P<0.01)$ in the percentage of inflammatory cells. The reduction of inflammatory cells after treatment with 5 ASA+PB was significantly greater $(P<0.01, P<0.05$, and $P<0.05$ for, respectively, CD3, CD4, and CD68) than after treatment with 5-ASA alone. Bars: dark blue, 5-ASA at the time of diagnosis; red, 5-ASA+PB at the time of diagnosis; green, 5-ASA after 6 months; violet, 5-ASA+PB after 6 months; light blue, normal mucosa (controls). 5-ASA indicates 5-aminosalicylic acid (mesalazine); PB, probiotics. $\frac{\text { full color }}{\ln l i n \mathrm{e}} \mid$ 


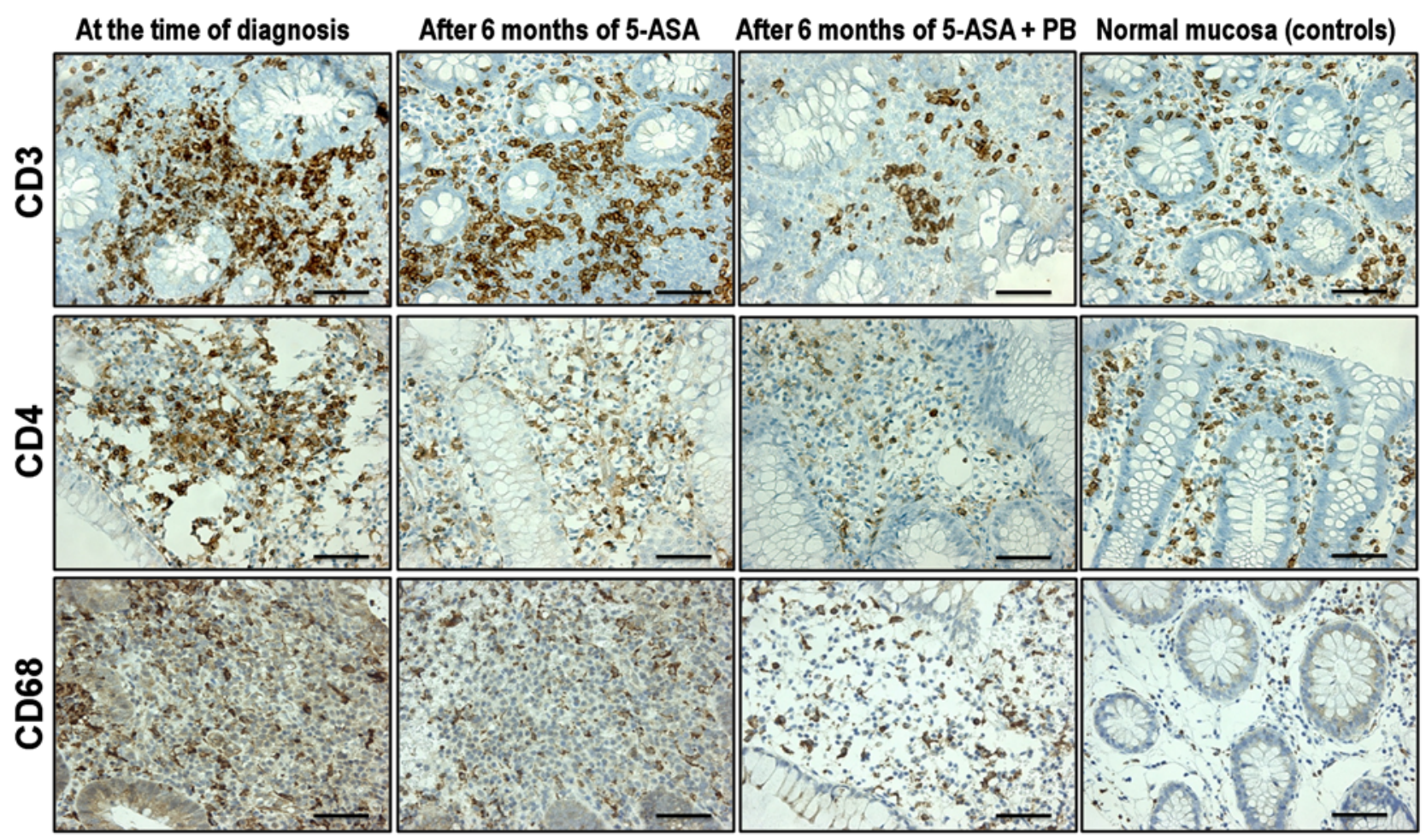

FIGURE 3. Representative images of inflammatory markers, that is, CD3-positive, CD4-positive, and CD68-positive cells, in colon mucosa. Positive cells are brown. Nuclei were counterstained with hematoxylin and appear blue. Bar: approximately $100 \mu \mathrm{m}$.

recognize the most relevant leukocytes, considering the objectives of our work (Table 1). All the molecular markers that we searched were expressed with variable percentages in all the examined tissues. We found significant variations only for CD3, CD4, and CD68 markers. Results are summarized in Table 2 and Figure 2. The percentages of these 3 molecular markers were highest at the time of diagnosis without differences between groups, but they decreased significantly after the treatments $(P<0.01)$. In addition, the differences between groups after treatment were significant $(P<0.01$ for CD3; $P<0.05$ for CD4; $P<0.05$ for CD68, Fig. 2). We also found a significant difference for the levels of CD3, CD4, and CD68 when both the treated groups were compared with normal mucosa (controls), with the exception of CD3 and CD4 when 5-ASA + PB patients were compared with controls (data not shown). Figure 3 shows a series of representative immunohistochemical pictures of these 3 markers. The greater numbers of positive cells were localized in lamina propria and only these were considered for our counts. These results demonstrated that the amelioration of clinical symptoms (see above) is associated with a reduction of inflammatory cells in colon mucosa, in agreement with the previous observations. ${ }^{28}$

\section{Immunohistochemistry of Hsp60}

To understand the mechanistic significance of Hsp60 in inflamed mucosa of patients with UC, we assessed the immunopositivity for Hsp60 separately in epithelium and lamina propria. Epithelial cells-responsible for mucosal permeability - represent a barrier to external stressors (eg, products of intestinal fermentation), whereas lamina propria - in which vessels and immune cells reside-is more susceptible to internal stressors (eg, inflammation). ${ }^{29-33}$ Results are summarized in Table 2 and Figure 4. Hsp60 positivity was localized in the cytoplasm and the greatest numbers of positive cells were consistently found in the epithelium. Moreover, levels of Hsp60 were consistently higher at the time of diagnosis (without differences between groups at this time) as compared with the sixth month end point, at which time a significant $(P<0.01)$ reduction in Hsp60 in the treated groups was evident. Interestingly, at the sixth month end point, we also found a significant difference between the 2 treated groups $(P<0.05$; Fig. 4$)$. Hsp60 expression in normal mucosa (controls) was significantly $(P<0.01)$ lower than in all the other groups. It is noteworthy that the regression analyses between Hsp60 and inflammatory markers showed a linear correlation between Hsp60 and CD68 levels in lamina propria (5ASA: $r=+0.504 ; P=0.0234$; 5-ASA + PB: $r=+0.809$; $P<0.0001$, Fig. 5) but not between Hsp60 and CD3 or CD4.

All together, these data support the view that Hsp60 is actively involved in UC pathogenesis as postulated earlier. ${ }^{5}$ We would also like to suggest that the increase of Hsp60 levels in relapsing patients is because of a stimulus (or stimuli), which is yet to be identified 

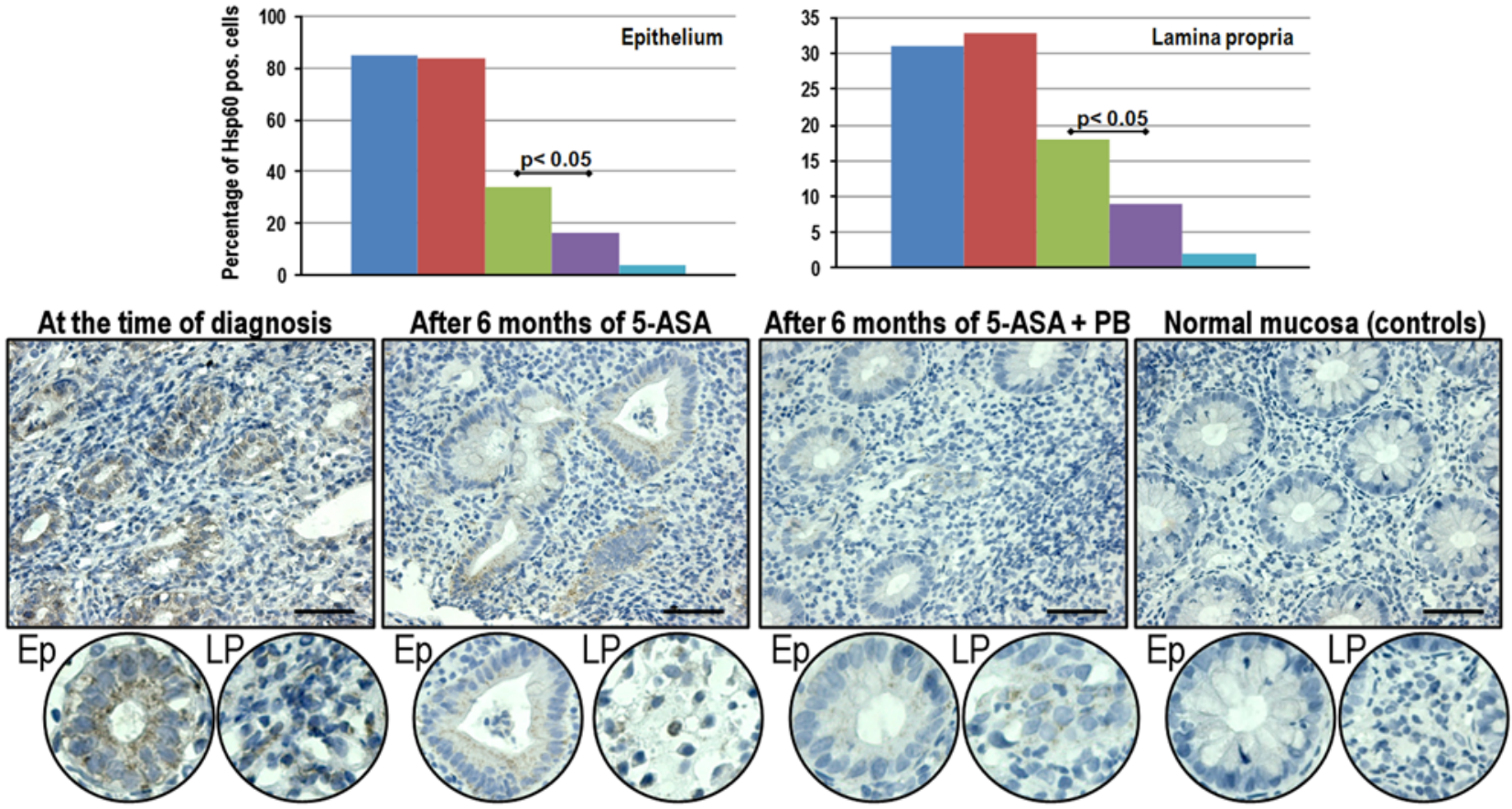

FIGURE 4. Immunohistochemical results and representative images of Hsp60-positive cells in colon mucosa. The top 2 panels display bars showing the levels of Hsp60-positive cells in epithelium and lamina propria. Both treatments determined a significant reduction of Hsp60 levels $(P<0.01)$. Moreover, Hsp60 reduction in 5-ASA+PB treated patients was significantly greater $(P<0.05)$ than in patients receiving 5-ASA alone. The middle panels show representative images of the immunohistochemical results for Hsp60. Positive cells are brown. Nuclei were counterstained with hematoxylin and appear blue. Bar: approximately $100 \mu \mathrm{m}$. The bottom circular panels show at a magnification higher than that in the middle panels the immunohistochemical images for Hsp60-positive cells in epithelium (Ep) and lamina propria (LP), demonstrating that the immunopositivity was localized in the cytoplasm. Top panel bars: dark blue, 5-ASA at the time of diagnosis; red, 5-ASA+PB at the time of diagnosis; green, 5-ASA after 6 months; violet, 5-ASA+PB after 6 months; light blue, normal mucosa (controls). 5-ASA indicates 5-aminosalicylic acid (mesalazine); Hsp, heat shock protein; PB, probiotics. full color

(mucosal cell stress induced by intestinal contents, including bacteria?) and, as Hsp60 levels consistently correlate with inflammation degree and disease severity, we think there is a cause-effect relationship between Hsp60 and pathology.

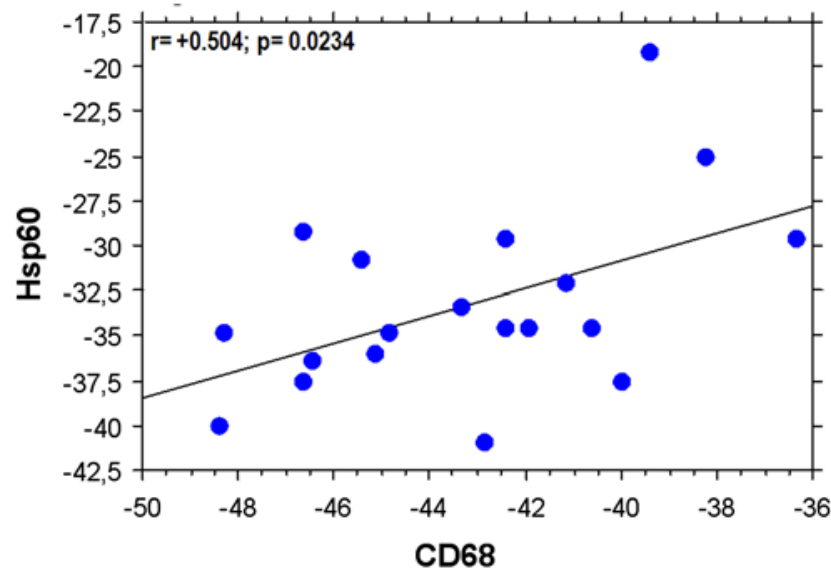

\section{Double Immunofluorescence of Hsp60 and CD68}

In view of the results described in the preceding paragraphs, and as it is known that CD68-positive macrophages have receptors for Hsp60, as TLR-4 ${ }^{34}$ and LOX-1, ${ }^{35}$

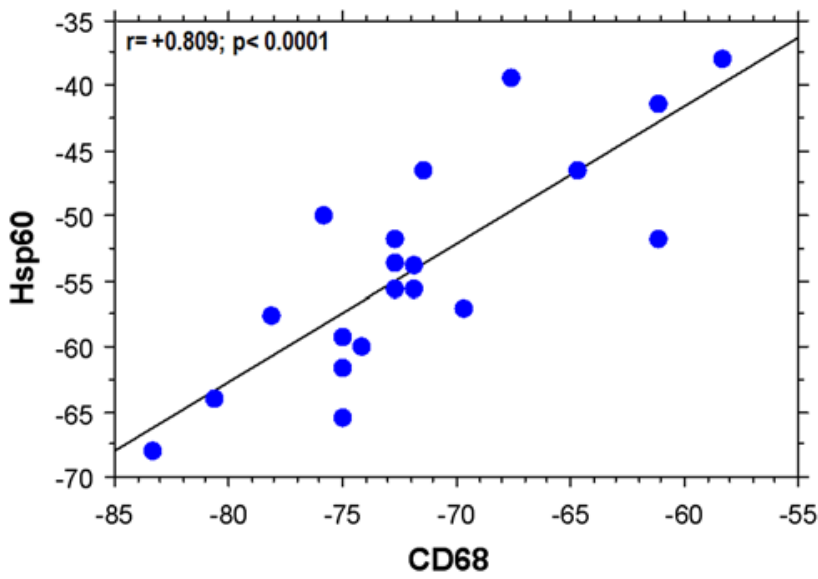

FIGURE 5. Hsp60-CD68 positive correlation. The regression plots show a significant positive correlation between Hsp60 and CD68 levels in both 5-ASA (left panel) and 5-ASA+PB (right panel) treated groups. Pertinent correlation coefficients (Pearson r) and " $P$ " values are indicated on top of each panel. 5-ASA indicates 5-aminosalicylic acid (mesalazine); Hsp, heat shock protein; PB, probiotics. full color 


\section{At the time of diagnosis}
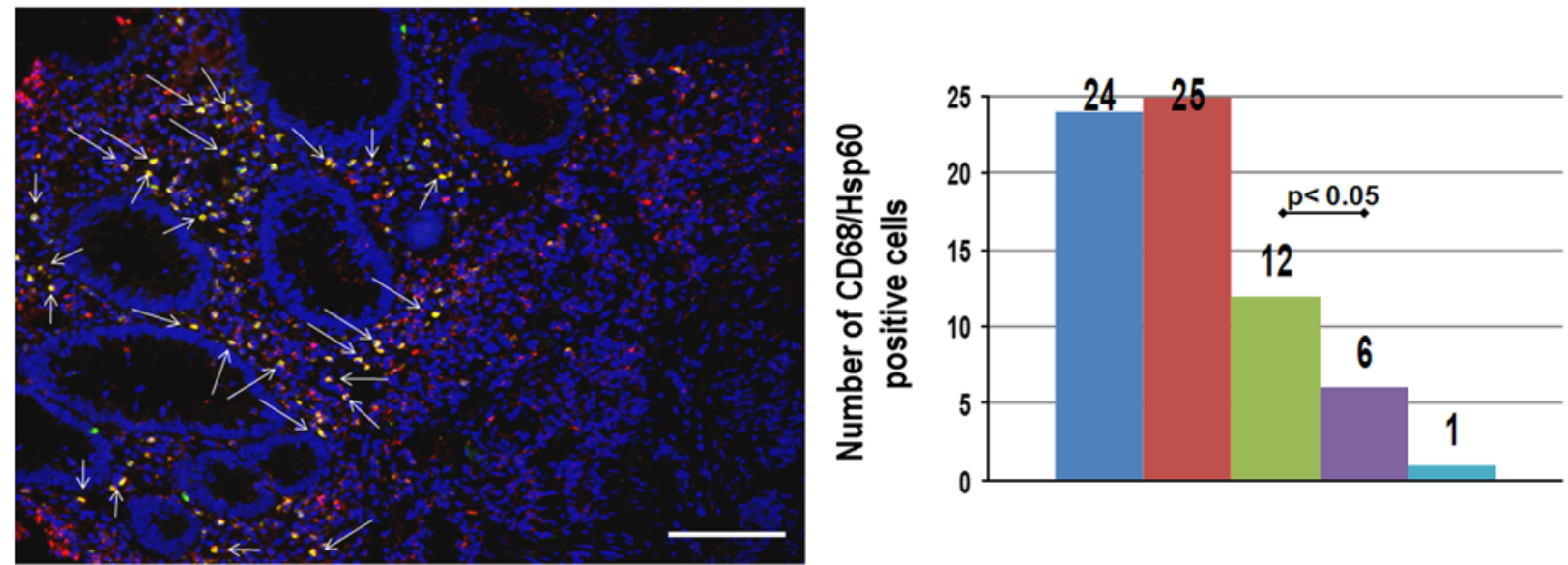

\section{After 6 months of 5-ASA}

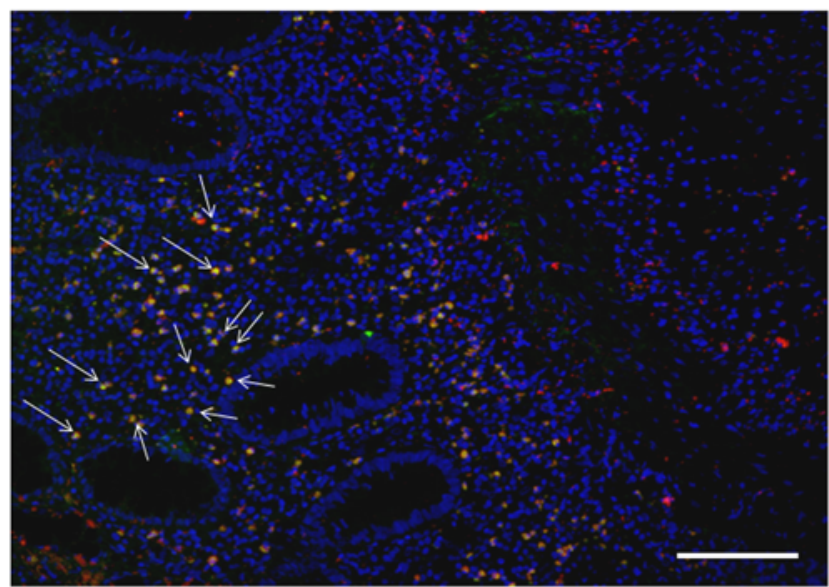

\section{After 6 months of 5-ASA + PB}

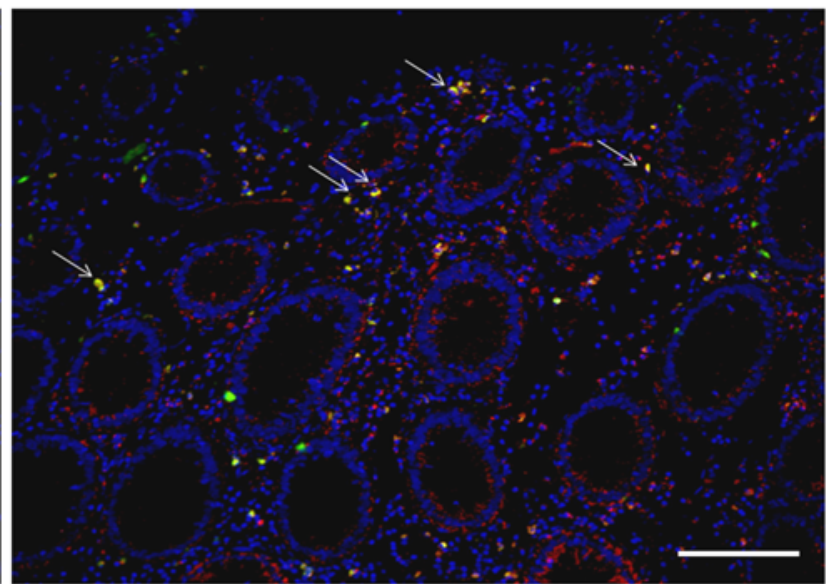

FIGURE 6. Double immunofluorescence results for Hsp60 and CD68 and representative images. The bars in the top right panel show the mean number of cells showing Hsp60-CD68 colocalization per high power field $(400 \times)$. This number was reduced significantly after both treatments $(P<0.01)$ and the reduction after 5 -ASA+PB treatment was significantly greater $(P<0.05)$ than after treatment with 5-ASA alone. The top left panel and the bottom panels contain illustrative images of the double immunofluorescence results for Hsp60 and CD68. Hsp60 appears red, CD68 appears green, and colocalization yellow/orange. Nuclei are counterstained with Hoechst and appear blue. Colocalization is indicated by arrows. Hsp60 and CD68 often colocalized in all specimens but the greatest numbers of cells showing Hsp60-CD68 colocalization were recorded in the samples taken before treatment. Bar: approximately $100 \mu \mathrm{m}$. Top right panel bars: dark blue, 5-ASA at the time of diagnosis; red, 5-ASA+PB at the time of diagnosis; green, 5-ASA after 6 months; violet, 5-ASA+PB after 6 months; light blue, normal mucosa (controls). 5-ASA indicates 5-aminosalicylic acid (mesalazine); Hsp, heat shock protein; PB, probiotics.

we decided to perform a double staining of these molecules to verify if they colocalize in the same cells. Results are summarized in Figure 6. Interestingly, most CD68 colocalized with Hsp60 in cells in all the examined slides, and this colocalization was most abundant at the time of diagnosis, before treatment. The number of cells in which colocalization of Hsp60 and CD68 occurred decreased significantly $(P<0.01)$ at the sixth month end point in the 2 treated groups, and this decrease was significantly more pronounced in the group that had received 5-ASA $+\mathrm{PB}$ than in the group that received only 5-ASA, as shown in Figure 6. The number of cells in which Hsp60 and CD68 colocalized was very low in colon mucosa (not shown). These data on colocalization of Hsp60-CD68 are in line with the immunohistochemical results described above, and support the view that Hsp60 stimulates the immune system via interaction with CD68positive cells, for example, macrophages and, in turn, amplifies the inflammatory reaction. ${ }^{36-38}$

\section{Immunoelectron Microscopy for Hsp60}

Although Hsp60 is considered a mitochondrial molecule, it has often been found in extramitochondrial sites, including cytosol and cell membrane, especially in pathologic conditions. ${ }^{16}$ To determine the intracellular localization of Hsp60 in our group of patients and controls, we resorted to immunoelectron microscopy. In both the treated groups at the time of diagnosis, Hsp60 was present both in mitochondria and cytosol, whereas 

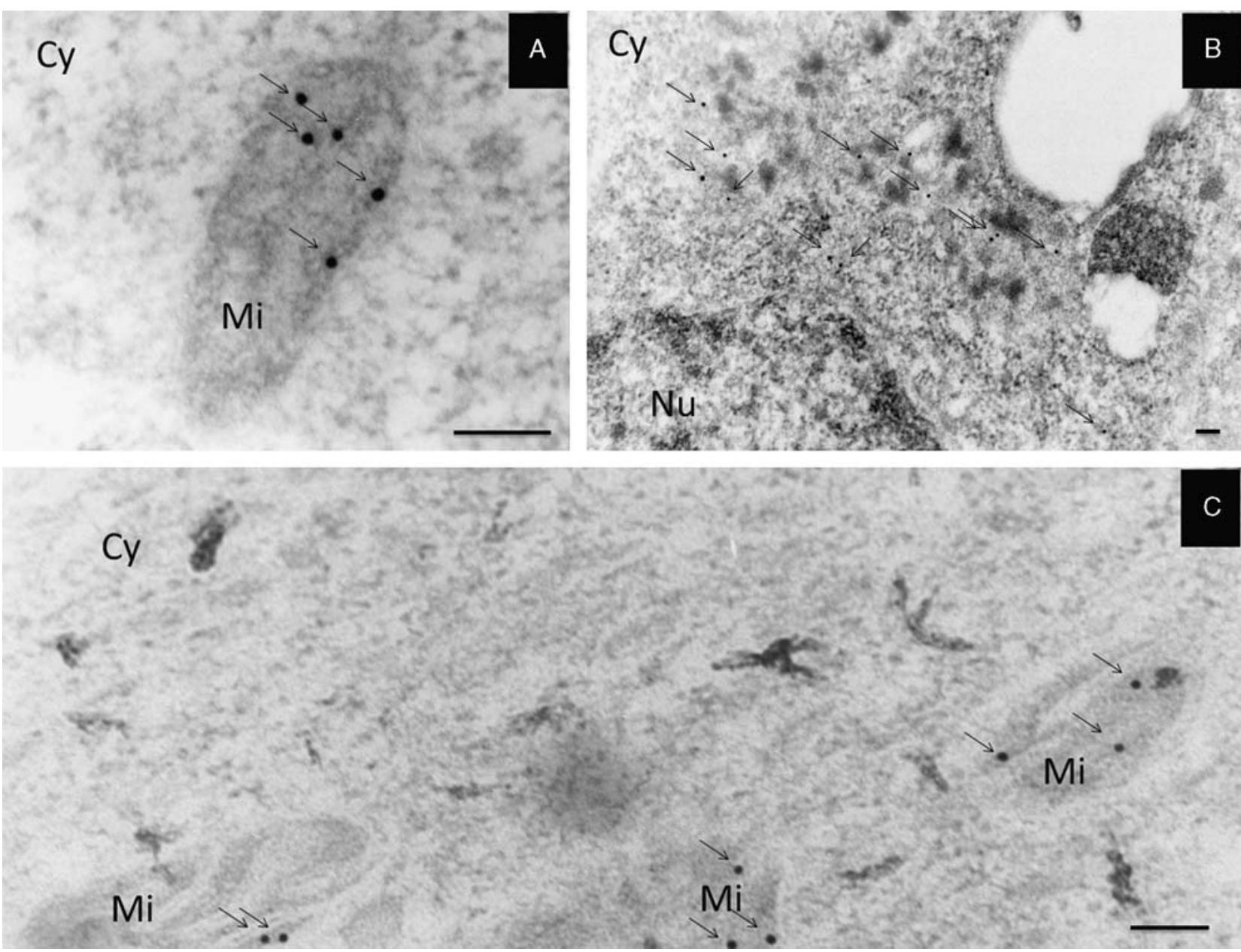

FIGURE 7. Immunoelectron microscopy for Hsp60. In these 3 images, representative of our results, the black spots indicated by arrows are $10 \mathrm{~nm}$ gold particles that show the presence of Hsp60. A, Control mucosa showing Hsp60 (arrows) inside a mitochondrium (Mi). Only the "shadow" of mitochondria can be seen in our preparations because the Karnofsky fixative we used for preservation of immunoreactivity of cells does not preserve their morphological details. B, Mucosal sample before treatment showing a diffuse Hsp60 localization (some indicated by arrows) in the cytosol (Cy). The nucleus (Nu) is also visible. C, Mucosal sample after treatment showing Hsp60 positivity (arrows) only in mitochondria. Hsp indicates heat shock protein. full color

after the treatment it was found only in mitochondria, as illustrated in Figure 7. These results are in agreement with the immunohistochemical data that showed reduced levels of Hsp60 after each of the 2 treatments, suggesting that the increased levels of Hsp60 before treatment were due to abnormally high levels of the chaperonin in the cytoplasm. As the presence of Hsp60 in the cytosol seems to be a prerequisite for its secretion, ${ }^{19,39}$ we would like to postulate that Hsp60 secretion occurs in relapsing UC patients but not (or at least not to the same extent) for those in remission. Reduced Hsp60 secretion would be directly related to amelioration of symptoms as less secreted Hsp60 would decrease Hsp60-macrophage interaction and, thus, reduce inflammation.

\section{CONCLUSIONS}

UC is a form of chronic, relapsing-remitting disease with an autoimmune component of unknown origin. ${ }^{40,41}$ Severity of symptoms may vary from patient to patient, but they include frequent episodes of diarrhea and rectal bleeding. ${ }^{42}$ Clinic assessment is done by the Mayo Score ${ }^{24}$ ranging from 0 to 12 . A score of 11 to 12 indicates a severe disease treated surgically or with biologic drugs. A lower score (4 to 10) indicates a mild-to-moderate disease often treated with 5-ASA, which results in long disease-free periods. ${ }^{42}$ More recently, a combined treatment with 5-ASA and probiotics demonstrated high efficacy in controlling symptoms and in extending the disease-free periods, ${ }^{24,43,44}$ although a placebo effect was also found. ${ }^{25}$

It has been postulated that, in UC, bacterial pathogens have a role in triggering inflammation and relapse. ${ }^{45,46}$ Commensal bacteria have a key role in preventing colonization by pathogens of the colon mucosa and, thus, in avoiding disequilibrium of intestinal flora and its consequence, stress of intestinal mucosa. ${ }^{47}$ This is the reason why we decided to compare the effects of treatments with either 5-ASA alone or in combination with a probiotic. 
Our results showed that in both the groups of patients, the treatment was effective to reduce symptoms and, thus, improve quality of life. This amelioration was associated with a reduction of inflammatory components, that is, CD3-positive, CD4-positive, and CD68-positive cells. Moreover, when we compared the results of the 2 types of treatment, 5-ASA versus the combination 5-ASA $+\mathrm{PB}$, we found a significant difference between the 2 groups for both clinical parameters and levels of inflammatory cells. Patients receiving the combined treatment responded better than those receiving only 5-ASA. Thus, the probiotics showed a definite clinical efficacy. This is in agreement with a number of other studies that showed efficacy of other probiotics in the treatment of IBD. ${ }^{25-28}$

In parallel, also Hsp60 levels were reduced after treatment (as shown by immunohistochemistry, double immunofluorescence, and immunoelectron microscopy) and this reduction was greater in patients treated with the combination 5-ASA + PB than in those receiving only 5ASA. Interestingly, the levels of Hsp60 were found to positively correlate with the levels of CD68, a marker of macrophages and dendritic cells. In addition, before and after the treatment, the great majority of CD68-positive cells in lamina propria were also positive for Hsp60. This is in agreement with the data from another laboratory ${ }^{48}$ showing that human Hsp60 is elevated in mononuclear cells in the mucosa of patients with IBD, a finding that prompted the authors to suggest that this chaperonin might play a role in the initiation or maintenance of the inflammatory process. It is known that CD68-positive macrophages have receptors for Hsp60, like TLR-2, TLR4, CD14, and LOX-1..$^{34,35}$ Thus, one may hypothesize that Hsp60 is taken up by CD68-positive cells via those receptors, something that ought to be tested experimentally. However, one cannot rule out that Hsp60 is produced by the macrophages or, even that both mechanisms, de novo synthesis of hsp60 by CD68-positive cells and binding by these cells of extracellular Hsp60 via surface receptors are at the basis of our observations on colocalization of Hsp60 with CD68. In case there was binding of Hsp60 via surface receptors, the expected consequence would be activation of CD68-positive cells followed by inflammatory response, as shown in the in vitro models. ${ }^{49-51}$ All together, these results support the working hypothesis that Hsp60 can play a decisive role in the pathogenesis of UC, and make Hsp60 a promising candidate for the treatment of UC with antichaperonin agents.

There is information in the literature suggesting that Hsp60 plays a pathogenetic role as autoantigen in some diseases with autoimmunity component, in which autoantibodies against the chaperonin may be present and probably participate in the mechanism of tissue damage. ${ }^{52-55}$ What we present here, in this work, is another, distinct aspect of Hsp60 involvement in pathogenesis. Our data pertain to a different mechanism in which Hsp60 does not act as autoantigen but as an immune-system signal factor that triggers components of the innate immune response, leading to inflammation rather than to antibody production. The 2 mechanisms are not mutually exclusive; on the contrary, both would contribute to initiation and progression of mucosal damage and to re-initiation of symptoms after a pause caused by treatment. However, the 2 mechanisms would call for different treatment approaches. If Hsp60 acts as autoantigen and anti-Hsp60 autoantibodies are pathogenetic factors, therapeutic strategies should include immune suppression. In contrast when Hsp60 acts as an inflammation-trigger factor, anti-inflammatory agents should be considered as well as Hsp60 blockers to avoid interaction of the chaperonin with its receptors on macrophages. There is however a point of contact between the 2 pathogenetic mechanisms, and that is the intestinal flora. It has been suggested that bacterial Hsp60 (GroEL) can invade the blood from its source in the intestine and induce anti-GroEL antibodies that will crossreact with the human Hsp60 and lead to autoimmune reactions in various tissues, including in the colon mucosa. ${ }^{56-58}$ Similarly, GroEL could penetrate the mucosal tissue and interact with local macrophages and initiate inflammation, adding to the effect of endogenous Hsp60 freed from stressed and damaged cells. Thus, management of the intestinal flora would be of the essence in the treatment of UC. Our data with probiotics would tend to support this hypothesis and lend support to treatment regimens that include improvement of the intestinal microbiota.

\section{REFERENCES}

1. Macario AJL, Lange M, Ahring BK, et al. Stress genes and proteins in the archaea. Microbiol Mol Biol Rev. 1999;63:923-967.

2. Kampinga $\mathrm{HH}$. Chaperones in preventing protein denaturation in living cells and protecting against cellular stress. Handb Exp Pharmacol. 2006;172:1-42.

3. Macario AJL, Conway de Macario E. Sick chaperones, cellular stress, and disease. $N$ Engl J Med. 2005;353:1489-1501.

4. Macario AJL, Conway de Macario E. Chaperonopathies and chaperonotherapy. FEBS Lett. 2007;581:3681-3688.

5. Rodolico V, Tomasello G, Zerilli M, et al. Hsp60 and Hsp10 increase in colon mucosa of Crohn's disease and ulcerative colitis. Cell Stress Chaperones. 2010;15:877-884.

6. Brocchieri L, Karlin S. Conservation among HSP60 sequences in relation to structure, function, and evolution. Protein Sci. 2000;9: 476-486.

7. Kreisel W, Hildebrandt H, Schiltz E, et al. Immuno-gold electron microscopical detection of heat shock protein 60 (hsp60) in mitochondria of rat hepatocytes and myocardiocytes. Acta Histochem. 1994;96:51-62.

8. Soltys BJ, Gupta RS. Immunoelectron microscopic localization of the $60-\mathrm{kDa}$ heat shock chaperonin protein (Hsp60) in mammalian cells. Exp Cell Res. 1996;222:16-27.

9. Gupta RS, Ramachandra NB, Bowes T, et al. Unusual cellular disposition of the mitochondrial molecular chaperones Hsp60, Hsp70 and Hsp10. Novartis Found Symp. 2008;291:59-68.

10. Lin KM, Lin B, Lian IY, et al. Combined and individual mitochondrial HSP60 and HSP10 expression in cardiac myocytes protects mitochondrial function and prevents apoptotic cell deaths induced by simulated ischemia-reoxygenation. Circulation. 2001;103: $1787-1792$.

11. Bross P, Naundrup S, Hansen J, et al. The Hsp60-(p.V98I) mutation associated with hereditary spastic paraplegia SPG13 compromises chaperonin function both in vitro and in vivo. $J$ Biol Chem. 2008;283:15694-15700.

12. Christensen $\mathbf{J H}$, Nielsen $\mathbf{M N}$, Hansen $\mathbf{J}$, et al. Inactivation of the hereditary spastic paraplegia-associated Hspdl gene encoding the 
Hsp60 chaperone results in early embryonic lethality in mice. Cell Stress Chaperones. 2010;15:851-863.

13. Hansen JJ, Dürr A, Cournu-Rebeix I, et al. Hereditary spastic paraplegia SPG13 is associated with a mutation in the gene encoding the mitochondrial chaperonin Hsp60. Am J Hum Genet. 2002;70: 1328-1332.

14. Magen D, Georgopoulos C, Bross P, et al. Mitochondrial hsp60 chaperonopathy causes an autosomal-recessive neurodegenerative disorder linked to brain hypomyelination and leukodystrophy. Am J Hum Genet. 2008;83:30-42.

15. Cappello F, Conway de Macario E, Marasà L, et al. Hsp60 expression, new locations, functions and perspectives for cancer diagnosis and therapy. Cancer Biol Ther. 2008;7:801-809.

16. Cappello F, Conway de Macario E, Di Felice V, et al. Chlamydia trachomatis infection and anti-Hsp60 immunity: the two sides of the coin. PLoS Pathog. 2009;5:e1000552.

17. Macario AJL, Cappello F, Zummo G, et al. Chaperonopathies of senescence and the scrambling of interactions between the chaperoning and the immune systems. Ann N Y Acad Sci. 2010;1197:85-93.

18. Lai HC, Liu TJ, Ting CT, et al. Regulation of IGF-I receptor signaling in diabetic cardiac muscle: dysregulation of cytosolic and mitochondria HSP60. Am J Physiol Endocrinol Metab. 2007; 292:E292-297.

19. Lin L, Kim SC, Wang Y, et al. HSP60 in heart failure: abnormal distribution and role in cardiac myocyte apoptosis. Am J Physiol Heart Circ Physiol. 2007;293:H2238-2247.

20. Chandra D, Choy G, Tang DG. Cytosolic accumulation of HSP60 during apoptosis with or without apparent mitochondrial release: evidence that its pro-apoptotic or pro-survival functions involve differential interactions with caspase-3. J Biol Chem. 2007;282: 31289-31301

21. Chun JN, Choi B, Lee KW, et al. Cytosolic Hsp60 is involved in the NF-kappaB-dependent survival of cancer cells via IKK regulation. PLoS One. 2010;5:e9422.

22. Cappello F, Bellafiore M, Palma A, et al. $60 \mathrm{KDa}$ chaperonin (HSP60) is over-expressed during colorectal carcinogenesis. Eur J Histochem. 2003;47:105-110.

23. Cappello F, David S, Rappa F, et al. The expression of HSP60 and HSP10 in large bowel carcinomas with lymph node metastase. $B M C$ Cancer. 2005;5:139.

24. Schroeder KW, Tremaine WJ, Ilstrup DM. Coated oral 5aminosalcylic acid therapy for mildly to moderately active ulcerative colitis. $N$ Eng $J$ Med. 1987;317:1625-1629.

25. Do VT, Baird BG, Kockler DR. Probiotics for maintaining remission of ulcerative colitis in adults. Ann Pharmacother. 2010;44:565-571.

26. Haller D, Antoine JM, Bengmark S, et al. Guidance for substantiating the evidence for beneficial effects of probiotics: probiotics in chronic inflammatory bowel disease and the functional disorder irritable bowel syndrome. J Nutr. 2010;140:690S-697S.

27. Reiff C, Kelly D. Inflammatory bowel disease, gut bacteria and probiotic therapy. Int J Med Microbiol. 2010;300:25-33.

28. MacDonald TT, Monteleone G, Pender SL. Recent developments in the immunology of inflammatory bowel disease. Scand J Immunol. 2000;51:2-9.

29. Keita AV, Söderholm JD. The intestinal barrier and its regulation by neuroimmune factors. Neurogastroenterol Motil. 2010;22:718-733.

30. Ohland CL, Macnaughton WK. Probiotic bacteria and intestinal epithelial barrier function. Am J Physiol Gastrointest Liver Physiol. 2010;298:G807-G819.

31. Sharma R, Young C, Neu J. Molecular modulation of intestinal epithelial barrier: contribution of microbiota. J Biomed Biotechnol. 2010;2010:305879.

32. Shimizu M. Interaction between food substances and the intestinal epithelium. Biosci Biotechnol Biochem. 2010;74:232-241.

33. Marchiando AM, Graham WV, Turner JR. Epithelial barriers in homeostasis and disease. Annu Rev Pathol. 2010;5:119-144.

34. Ohashi K, Burkart V, Flohé S, et al. Cutting edge: heat shock protein 60 is a putative endogenous ligand of the toll-like receptor-4 complex. J Immunol. 2000;164:558-561.

35. Xie J, Zhu H, Guo L, et al. Lectin-like oxidized low-density lipoprotein receptor-1 delivers heat shock protein 60 -fused antigen into the MHC class I presentation pathway. J Immunol. 2010;185: 2306-2313.

36. van Eden W. Immunoregulation of autoimmune diseases. Hum Immunol. 2006;67:446-453.

37. Alard JE, Dueymes M, Youinou P, et al. HSP60 and anti-HSP60 antibodies in vasculitis: they are two of a kind. Clin Rev Allergy Immunol. 2008;35:66-71

38. Fischer B, Elias D, Bretzel RG, et al. Immunomodulation with heat shock protein DiaPep277 to preserve beta cell function in type 1 diabetes-an update. Expert Opin Biol Ther. 2010;10:265-272.

39. Merendino AM, Bucchieri F, Campanella C, et al. Hsp60 is actively secreted by human tumor cells. PLoS One. 2010;5:e9247.

40. Szigethy E, McLafferty L, Goyal A. Inflammatory bowel disease. Child Adolesc Psychiatr Clin N Am. 2010;19:301-318.

41. Engel MA, Neurath MF. New pathophysiological insights and modern treatment of IBD. J Gastroenterol. 2010;45:571-583.

42. Vecchi M, Saibeni S, Devani M, et al. Review article: diagnosis, monitoring and treatment of distal colitis. Aliment Pharmacol Ther 2003;17(suppl 2):2-6.

43. Dharmani P, Chadee K. Biologic therapies against inflammatory bowel disease: a dysregulated immune system and the cross talk with gastrointestinal mucosa hold the key. Curr Mol Pharmacol. 2008; $1: 195-212$

44. Sang LX, Chang B, Zhang WL, et al. Remission induction and maintenance effect of probiotics on ulcerative colitis: a metaanalysis. World J Gastroenterol. 2010;16:1908-1915.

45. Andoh A, Fujiyama Y. Therapeutic approaches targeting intestinal microflora in inflammatory bowel disease. World $J$ Gastroenterol. 2006; 12:4452-4460

46. Arnett HA, Viney JL. Gatekeepers of intestinal inflammation. Inflamm Res. 2010;59:1-14.

47. Elson CO, Cong Y, Iqbal N, et al. Immuno-bacterial homeostasis in the gut: new insights into an old enigma. Semin Immunol. 2007;13: 187-194.

48. Peetermans WE, D'Haens GR, Ceuppens JL, et al. Mucosal expression by $\mathrm{B} 7$-positive cells of the 60-kilodalton heat-shock protein in inflammatory bowel disease. Gastroenterology. 1995;108:75-82.

49. Habich C, Baumgart K, Kolb H, et al. The receptor for heat shock protein 60 on macrophages is saturable, specific, and distinct from receptors for other heat shock proteins. J Immunol. 2002;168: 569-576.

50. Habich C, Kempe K, van der Zee R, et al. Different heat shock protein 60 species share pro-inflammatory activity but not binding sites on macrophages. FEBS Lett. 2003;533:105-109.

51. Habich C, Kempe K, Gomez FJ, et al. Heat shock protein 60 identification of specific epitopes for binding to primary macrophages. FEBS Lett. 2006;580:115-120.

52. Handley HH, Yu J, Yu DT, et al. Autoantibodies to human heat shock protein (hsp)60 may be induced by Escherichia coli groEL. Clin Exp Immunol. 1996;103:429-435.

53. Yokota SI, Hirata D, Minota S, et al. Autoantibodies against chaperonin CCT in human sera with rheumatic autoimmune diseases: comparison with antibodies against other Hsp60 family proteins. Cell Stress Chaperones. 2000;5:337-346.

54. Prohászka Z, Duba J, Horváth L, et al. Comparative study on antibodies to human and bacterial $60 \mathrm{kDa}$ heat shock proteins in a large cohort of patients with coronary heart disease and healthy subjects. Eur J Clin Invest. 2001;31:285-292.

55. Shovman O, Sherer Y, Gilbourd B, et al. Low levels of heat shock proteins-60 and -65 autoantibodies in Sjögren's syndrome. Isr Med Assoc J. 2005;7:778-780.

56. Stevens TR, Winrow VR, Blake DR, et al. Circulating antibodies to heat-shock protein 60 in Crohn's disease and ulcerative colitis. Clin Exp Immunol. 1992;90:271-274.

57. Bene L, Füst G, Huszti Z, et al. Impaired humoral immune response against mycobacterial $65-\mathrm{kDa}$ heat shock protein (HSP65) in patients with inflammatory bowel disease. Dig Dis Sci. 2002;47: 1432-1437.

58. Huszti Z, Bene L, Kovács A, et al. Low levels of antibodies against $E$. coli and mycobacterial $65 \mathrm{kDa}$ heat shock proteins in patients with inflammatory bowel disease. Inflamm Res. 2004;53:551-5555. 